\title{
Protocolo de enfermagem para o cuidado da pessoa com diabetes mellitus na atenção primária
}

Nursing protocol for the care of people with diabetes mellitus in primary care

Protocolo de enfermería para la atención de personas con diabetes mellitus en atención primaria

\section{Priscylla Lauterte ${ }^{\mathrm{I}}$, Denise Maria Vieira Guerreiro da Silva ${ }^{\mathrm{II}}$, Maria Aparecida Salci ${ }^{\mathrm{III}}$, Ivonete Teresinha Schuelter Buss Heidemann ${ }^{\text {IV }}$, Priscila Juceli Romanoskiv}

Resumo: Objetivo: avaliar a contribuição do Protocolo de Enfermagem -Volume I, para o cuidado à saúde de pessoas com diabetes mellitus tipo 2 na perspectiva de enfermeiros e médicos que atuam na Atenção Primária à Saúde. Método: pesquisa avaliativa qualitativa, realizada com 22 profissionais, sendo 19 enfermeiros e três médicos, no período de junho a agosto de 2017, utilizando entrevistas semiestruturadas e análise de dados na perspectiva de conteúdo convencional. Resultados: evidenciou-se que o Protocolo foi implantado de forma organizada e sistemática, com foco no cuidado integral e alinhamento entre médicos e enfermeiros por meio da interconsulta. Promoveu a ampliação do acesso à saúde das pessoas com diabetes oferecendo autonomia, respaldo e resolutividade aos enfermeiros. Conclusão: o Protocolo contribuiu para a qualificação do cuidado de enfermagem às pessoas com diabetes, com reconhecimento e confiança dos enfermeiros em relação à qualidade do conteúdo, pertinência e aplicabilidade.

Descritores: Protocolos; Enfermeiras e enfermeiros; Diabetes mellitus; Cuidados de enfermagem; Atenção primária à saúde

Abstract: Objective: to evaluate the contribution of the Nursing Protocol - Volume I, for the health care of people with type 2 diabetes mellitus from the perspective of nurses and doctors working in Primary Health Care. Method:

\footnotetext{
${ }^{\text {I }}$ Priscylla Lauterte. Enfermeira. Mestre em Enfermagem. Secretaria Municipal de Saúde de Florianópolis, Florianópolis/SC, Brasil. e-mail: pry.enfermeira@gmail.com ORCID: https://orcid.org/0000-0002-4855-2053

IIII Denise Maria Guerreiro Vieira da Silva. Enfermeira, Doutora em Enfermagem, Universidade Federal de Santa Catarina, Florianópolis/SC, Brasil. e-mail: denise_guerreiro@hotmail.com. ORCID: https://orcid.org/0000-0003-2139-083X.

III Maria Aparecida Salci. Enfermeira, Doutora em Enfermagem, Universidade Estadual de Maringá, Maringá/PR, Brasil. e-mail: masalci@uem.br. ORCID: http://orcid.org/0000-0002-6386-1962

IV Ivonete Teresinha Schulter Buss Heidemann. Enfermeira, Doutora em Enfermagem, Universidade Federal de Santa Catarina, Florianópolis/SC, Brasil. e-mail: ivonete.heidemann@ufsc.br ORCID: https://orcid.org/0000-0001-6216-1633

${ }^{v}$ Priscila Juceli Romanoski. Enfermeira. Doutoranda em Enfermagem. Universidade Federal de Santa Catarina, Florianópolis/SC, Brasil. e-mail: priscila.romanoski@gmail.com ORCID: https://orcid.org/0000-0002-4494-9897
} 
qualitative evaluative research, carried out with 22 professionals, including 19 nurses and three doctors, from June to August 2017, using semi-structured interviews and data analysis from the perspective of conventional content. Results: it became evident that the Protocol was implemented in an organized and systematic manner, with a focus on comprehensive care and alignment between doctors and nurses through inter-consultation. It promoted the expansion of access to health for people with diabetes, offering autonomy, support and resolution to nurses. Conclusion: the Protocol contributed to the qualification of nursing care for people with diabetes, with nurses' recognition and confidence in relation to the quality of content, relevance and applicability.

Descriptors: Protocols; Nurses and nurses; Diabetes mellitus; Nursing care; Primary health care

Resumen: Objetivo: evaluar la contribución del Protocolo de Enfermería - Volumen I, para la atención de la salud de las personas con diabetes mellitus tipo 2 desde la perspectiva de los enfermeros y médicos que trabajan en Atención Primaria de Salud. Método: investigación evaluativa cualitativa, realizada con 22 profesionales, 19 enfermeras y tres médicos, de junio a agosto de 2017, mediante entrevistas semiestructuradas y análisis de datos desde la perspectiva de contenido convencional. Resultados: se evidenció que el Protocolo se implementó de manera organizada y sistemática, con foco en la atención integral y la alineación entre médicos y enfermeras a través de la interconsulta. Promovió la expansión del acceso a la salud para las personas con diabetes, ofreciendo a los enfermeros autonomía, apoyo y resolución. Conclusión: el Protocolo contribuyó a la calificación de la atención de enfermería a las personas con diabetes, con el reconocimiento y la confianza de las enfermeras en relación a la calidad del contenido, relevancia y aplicabilidad.

Descriptores: Protocolos; Enfermeras y enfermeros; Diabetes mellitus; Cuidado de enfermería; Primeros auxilios

\section{Introdução}

Diabetes mellitus tipo 2 (DM2) é, segundo a Sociedade Brasileira de Diabetes, um distúrbio metabólico caracterizado por hiperglicemia persistente, decorrente de deficiência na produção de insulina ou na sua ação, ou em ambos os mecanismos. ${ }^{1}$ A atenção às pessoas com DM requer uma atuação multiprofissional, com destaque para o enfermeiro, que deve realizar atividades educativas e de cuidado, incluindo consulta de enfermagem, solicitação de exames e renovação de medicamentos, sendo que esses dois últimos, desde que estabelecidos em protocolos municipais. $^{2}$

A utilização de protocolos de Enfermagem na atenção às pessoas com DM2 é reconhecida como fundamental para a melhoria da saúde dessa população, trazendo efeitos positivos no cuidado. ${ }^{1}$ O enfermeiro tem como atribuições de destaque, o desenvolvimento de atividades educativas e de capacitação de sua equipe na atenção à essas pessoas. ${ }^{2}$ 
A proposta de criação de protocolos assistenciais é fundamentada no reconhecimento de que os mesmos mostram vantagens, tais como: promover maior segurança aos profissionais e usuários; ações de cuidados mais uniformes entre os profissionais; melhoria na tomada de decisões; incorporação de novas tecnologias; uso racional de recursos; disseminação de conhecimento; comunicação profissional; e coordenação do cuidado. Sendo construídos baseados nas melhores e mais atuais evidências científicas, tratando-se de instrumentos legais de uso clínico. ${ }^{3-4}$

Nesse contexto, a Comissão de Sistematização da Assistência de Enfermagem da Secretaria Municipal de Saúde de Florianópolis/Santa Catarina (SC) elaborou e publicou em 2015, o Protocolo de Enfermagem em hipertensão, diabetes e outros fatores associados a doenças cardiovasculares - Volume I. Esse Protocolo foi e vem sendo atualizado sistematicamente pelos enfermeiros que compõem a refeirda comissão, a qual elaborou posteriormente outros cinco protocolos em diferentes áreas de atuação da enfermagem. A elaboração do Protocolo foi decorrente do reconhecimento da necessidade de dar maior autonomia aos enfermeiros no cuidado às pessoas com DM2 e hipertensão arterial, bem como trazer segurança e respaldo legal em suas condutas na Atenção Primária à Saúde (APS). Esse Protocolo amplia a atuação clínica do enfermeiro, especialmente em condutas que eram, até o momento, realizadas exclusivamente pelos médicos, como a solicitação de exames e atualização de prescrição de medicamentos.

A criação de Protocolos de Enfermagem contribui para a sistematização da assistência, trazendo qualificação para o cuidado prestado à população. No entanto, mesmo com a presença desses instrumentos implantados em algumas instituições, ainda são escassas as pesquisas sobre o tema. ${ }^{6-7}$ Nesse sentido, é essencial que haja um acompanhamento e avaliação do processo de implementação, de forma a consolidar e/ou reorientar a prática de uso dos protocolos. 
Protocolo de enfermagem para o cuidado da pessoa com diabetes mellitus na atenção P... | 4

Diante desse contexto, a questão de pesquisa que conduziu o estudo foi "Qual a contribuição do Protocolo de Enfermagem - Volume I da Secretaria Municipal de Saúde de Florianópolis para o cuidado à saúde das pessoas com DM2 atendidas na APS?” e o objetivo da pesquisa foi avaliar a contribuição do Protocolo de Enfermagem - Volume I, para o cuidado à saúde de pessoas com DM2 na perspectiva de enfermeiros e médicos que atuam na APS.

\section{Método}

Trata se de uma pesquisa avaliativa qualitativa. Para efetuar essa avaliação, utilizou-se como referência teórica a Política Nacional de Atenção Básica, os princípios e diretrizes: universalidade, resolutividade, longitudinalidade, coordenação do cuidado, ordenamento das redes e participação da comunidade. ${ }^{8}$

O foco da avaliação foi o capítulo dois do Protocolo de Enfermagem - Volume I que se refere ao cuidado às pessoas com DM2, devido a sua relevância na saúde pública. O conteúdo do capítulo inclui: solicitação de glicemia em jejum; renovação de medicamentos; solicitação de exames de acompanhamento de pessoas que já possuem diagnóstico de diabetes; avaliação geral da pessoa com diabetes, incluindo avaliação dos pés; orientação quanto ao tratamento medicamentoso.

O estudo foi realizado no município de Florianópolis/SC com 22 profissionais, sendo 19 enfermeiros e três médicos que atuavam em 10 Centros de Saúde (CS) dos quatro Distritos Sanitários do município. Foram selecionados os CSs em que os enfermeiros tinham incorporado a prática do uso do Protocolo de Enfermagem em Hipertensão, Diabetes e outros fatores associados a doenças cardiovasculares - Volume I, desde a implementação do mesmo, que iniciou no mesmo ano de sua publicação, em 2015. Essa informação foi obtida pela gestão que acompanha a implantação do Protocolo nos CSs. 
A seleção dos enfermeiros foi intencional, atendendo os seguintes critérios de inclusão: ter vínculo empregatício com a Secretaria Municipal de Saúde; estar realizando atenção em saúde às pessoas com DM; e, estar utilizando o Protocolo de Enfermagem - Volume I no cuidado às pessoas com DM desde sua implementação. Uma primeira informação acerca de quem poderia ser incluído no estudo foi obtida com o setor de gerência da APS e confirmada com o profissional ao convidá-lo a participar do estudo. Nesse sentido, fizeram parte do estudo os profissionais que indicaram estar utilizando o Protocolo de forma efetiva e ter conhecimento sobre o mesmo.

A inclusão dos médicos foi por indicação dos enfermeiros que estavam trabalhando com esses profissionais na atenção à pessoa com DM, desde que atendidos os critérios de seleção: ter vínculo empregatício com a Secretaria Municipal de Saúde; estar realizando o compartilhamento do cuidado da pessoa com diabetes com o enfermeiro. Todos os convites para médicos e enfermeiros foram feitos verbalmente e dentre os que atendiam aos critérios de inclusão, não houve recusa dos enfermeiros, porém dois médicos não aceitaram participar, indicando falta de tempo.

A coleta de dados ocorreu entre junho a agosto de 2017 e a técnica adotada foi a entrevista individual que foi guiada por um roteiro semiestruturado, com duração média de 35 minutos. Foram gravadas, com consentimento dos entrevistados, em equipamento de captação de áudio digital. As entrevistas tiveram como questões básicas: Conte-me um pouco sobre sua trajetória na enfermagem ou medicina e a escolha por trabalhar na atenção primária? Como é a atenção às pessoas com DM neste CS? Qual sua percepção sobre o uso dos protocolos de Enfermagem na APS?

As gravações das entrevistas foram transcritas e a análise dos dados se deu por meio da análise qualitativa de conteúdo convencional ${ }^{9}$ o que gerou uma codificação de 230 códigos 
Protocolo de enfermagem para o cuidado da pessoa com diabetes mellitus na atenção P... 6

organizados por semelhança resultando em duas categorias: Ampliação do cuidado de enfermagem e Segurança da atuação do enfermeiro no cuidado às pessoas com DM.

Foram respeitados os aspectos éticos e legais da Resoluções 466/2012 e 510/2016, que aprovam as diretrizes e normas regulamentadoras de pesquisa envolvendo os seres humanos, do Conselho Nacional de Saúde. O estudo teve aprovação do Comitê de Ética e Pesquisa com Seres Humanos em 15 de maio de 2017 sob № 2.064.389. Todos os participantes assinaram o Termo de Consentimento Livre e Esclarecido em duas vias. No intuito de preservar o anonimato os enfermeiros foram identificados por meio de um sistema numérico (E1 a E19) e os médicos (Em1 a Em3).

\section{Resultados}

Dentre os 22 participantes da investigação, 17 eram do sexo feminino e cinco do sexo masculino, com idade entre 26 e 48 anos. O tempo de formação variou entre quatro e 36 anos, e o tempo de atuação desses profissionais na APS variou entre dois e 23 anos.

De modo geral, o Protocolo de Enfermagem foi avaliado pelos participantes como um documento que trouxe melhorias para o serviço de saúde, contribuído com o processo de trabalho, possibilitando renovação e qualificação nesse atendimento, oferecendo autonomia, respaldo e resolutividade ao enfermeiro, no cuidado às pessoas com DM na APS.

\section{Ampliação do cuidado de enfermagem}

O Protocolo implementado na APS de Florianópolis/SC possibilitou ao enfermeiro condutas mais abrangentes, como a solicitação de exames para investigação clínica, rastreamento do DM e renovações de receitas de medicamentos de uso contínuo, trazendo assim, agilidade no atendimento. O profissional tornou-se mais autônomo em sua prática, 
permitindo que conseguisse atuar nas demandas de saúde mais comuns no seu cotidiano de trabalho.

Eu acho que o Protocolo permite uma autonomia ao enfermeiro e tu vai conseguir ampliar esse acesso, ampliar o cuidado do paciente. Pelo menos eu vi em outros municípios que a atuação do enfermeiro é muito restrita, e aí todo esse acompanhamento do paciente, a longitudinalidade, tudo isso se perde quando o enfermeiro não está inserido no cuidado. Então, o Protocolo traz esse respaldo, esse aporte teórico e faz com que o enfermeiro consiga atuar de uma forma bem mais autônoma, desvinculada, muitas vezes, do médico naquele momento de consulta, ele começa e finaliza o atendimento sozinho. (E06)

O Protocolo também oportunizou o fortalecimento de atribuições que já eram realizadas pelo enfermeiro, como a educação em saúde, a prevenção do pé diabético e a prevenção de complicações. A avaliação sistemática do pé das pessoas com DM foi considerada um avanço importante no cuidado de enfermagem, uma vez que anteriormente essas avaliações eram eventuais ou mesmo, não realizadas.

Porque os médicos não fazem exame dos pés, não têm tempo. Então, conseguimos pegar esses pacientes que talvez, a grande maioria nunca tenha feito. A gente sabe a importância do que é isso, que um exame tão simples, barato e rápido, pode evitar complicação na vida de uma pessoa. (E01)

Dentro dessa prática de uso do Protocolo, houve uma ampliação do acesso do enfermeiro às pessoas com DM, pela possibilidade de mais um profissional atuar com vistas à integralidade e reconhecimento da resolutividade no atendimento. Nesse sentido houve fortalecimento de alguns atributos da APS, na visão dos enfermeiros e médicos entrevistados.

Eu acho que, principalmente na questão do acesso, se tu amplias o cuidado de enfermagem, tu dá mais respaldo para o enfermeiro atuar. Caso contrário, não conseguiríamos atender a demanda espontânea, aumentaria as filas de pacientes, não teria espaço na agenda. Antes dos Protocolos, tínhamos muita dificuldade nessa questão do acesso, claro 
Protocolo de enfermagem para o cuidado da pessoa com diabetes mellitus na atenção P... $\mid 8$

que a gente acabava fazendo, mas com a interconsulta, tendo que ter o respaldo do médico em todas as situações. Hoje temos independência para atuar. (E11)

Eu acho que no acesso por ele aumentar a resolutividade da enfermagem, isso amplia o acesso porque você vai ter mais de um profissional capacitado para atender esses pacientes, então acaba que a gente consegue atender um número maior de pessoas e isso amplia o acesso. $O$ vínculo a partir do momento que a pessoa começa a passar mais tanto com o médico quanto com o enfermeiro ele acaba criando um vínculo porque ele tem mais contato com esse profissional. (Em2)

Os médicos que trabalham com os enfermeiros que incorporaram o Protocolo em sua prática reconheceram a melhora no acompanhamento de pessoas com DM, percebendo também um fortalecimento do compartilhamento de ações com o enfermeiro.

Olha, o que eu sei é que aumentou a resolutividade da enfermagem. Antes várias coisas que o enfermeiro não poderia fazer, agora ele pode fazer. Então, melhorou o trabalho em equipe, o compartilhamento de funções. (Em2)

Agora o papel do Protocolo é essencial, porque primeiro aquela demanda de muitas coisas que não precisam ser necessariamente médicocentristas, e outras coisas que até a enfermagem tem um potencial maior do que a gente. (Em1)

Apesar dessa preponderância no reconhecimento dos aspectos positivos do Protocolo para a atenção às pessoas com DM, também foram identificadas algumas fragilidades nessa implantação. Os enfermeiros reconheceram que uma demanda excessiva pode prejudicar a utilização do Protocolo.

Eu acho que uma dificuldade é o fato dos enfermeiros estarem sobrecarregados, com uma pressão de demanda imensa. (E02)

Outro aspecto que tem trazido dificuldades é o fato da rede privada de farmácias não aceitar a prescrição de medicamentos pelo enfermeiro. Essa situação ocorre quando não há o 
medicamento disponível no CS e a pessoa precisa adquiri-lo em farmácias particulares, caso não haja alternativa terapêutica.

Apesar de algumas dificuldades, foi apresentada pelos enfermeiros a valorização de ações possibilitadas a eles pelo uso do Protocolo, na perspectiva da ampliação da clínica e qualificação do cuidado às pessoas com DM.

\section{Segurança no cuidado às pessoas com diabetes mellitus}

Os enfermeiros, após a implementação do Protocolo, sentem-se empoderados para assumir práticas que, anteriormente, eram realizadas somente pelo médico. O Protocolo lhes dá confiança, por manter uma dinâmica de atualização e de possibilidade de revisão a partir de novas evidências. Além disso, consideram que desenvolver atividades como a prescrição de medicamentos e a solicitação de exames é o reconhecimento da boa formação do enfermeiro para tais atividades clínicas.

Os enfermeiros identificaram o Protocolo, desde o início de sua implantação, como sendo um documento claro e explicativo, enxuto e objetivo, estando online e em formato PDF, facilitando seu uso em sua prática diária. A forma objetiva como o Protocolo está apresentado viabiliza seu uso durante os atendimentos.

O protocolo é ótimo, porque a gente tem conhecimento dos cadernos de atenção do Ministério, mas é difícil tu parar e ler tudo, pois são grandes. Então, eu acho que o protocolo do município veio para nos respaldar, ampliando a nossa assistência, fazendo com que sejamos mais resolutivos em alguns casos, e ele é bem enxuto. (E01)

Mesmo que inicialmente tenham se sentido um pouco inseguros com o uso do Protocolo, especialmente a renovação de receitas de medicamentos, com o seu uso mais rotineiro, passaram a reconhecer as facilidades que ele traz na atenção às pessoas com DM. Porém, 
Protocolo de enfermagem para o cuidado da pessoa com diabetes mellitus na atenção P... $\mid 10$

reconhecem e sugerem que a educação continuada deva ser institucionalizada no município, contribuindo ainda mais com a formação do profissional.

Devemos investir muito em capacitação permanente, porque sempre vai ter gente nova, sempre vai ter dúvida, ter gente que esqueceu, ter coisa para atualizar. (E02)

Olha eu acho que um grande desafio é a gente ter tempo, todo mundo, a equipe inteira, ter tempo de parar para ler, estudar, o protocolo novo, porque é uma informação nova, então precisa de tempo para leitura, para estudar. E acho que um desafio é que é saber, porque tem as coisas novas que podem e tem coisas que não podem então lembrar de tudo, assim, o que pode e o que não pode eu percebo que é uma dificuldade. (Em2)

A padronização das condutas que o Protocolo estabelece, dá a certeza de que estão incluindo todos os cuidados básicos necessários, mas ainda mantém sua autonomia nas decisões relacionadas a cada situação específica.

[...] é legal até como estímulo para o colega e para valorizar nossa profissão, sabe? Porque acho que o Protocolo, ele veio muito para valorizar o saber do enfermeiro, os treinamentos e a formação que a gente teve. Isso é muito nosso, é da nossa classe, da nossa categoria de firmar isso, de dizer: "sim, a universidade nos capacitou. Sim, eu me sinto segura para isso!”. (E08)

Eu posso te dizer que a gente tem modificado muito a nossa dinâmica de trabalho, o protocolo veio em parte a gente tem utilizado não só o de diabetes, para outras coisas tem muitas coisas que as vezes no próprio atendimento para acolher a demanda espontânea, a enfermagem vem discute um caso e quando se enquadra no que está no protocolo, as vezes elas discutem alguma dúvida conosco elas vão lá e prescrevem aquela situação. (Em3)

Para auxiliar na expansão da implantação do Protocolo, no Estado foram realizadas capacitações, ministradas por enfermeiros da Secretaria Municipal de Saúde com apoio do Conselho Regional de Enfermagem de SC para outros município estimulando a adesão em 
11 | Lauterte P, Silva DMVG, Salci MA, Heidemann ITSB, Romanoski PJ

outras localidades. Isso ocorreu pois a implantação do Protocolo, por ser pioneiro e uma novidade na rotina profissional, trouxe algumas inseguranças pela dificuldade de troca de experiência.

E aí a sugestão é de pensar na atuação do enfermeiro em outros países, por exemplo, em outros locais onde a gente sabe que a atuação do enfermeiro é mais ampla do que aqui no Brasil. A gente sabe que no Brasil, Florianópolis tem sido pioneiro nisso. Então, isso eu entendo que gera uma insegurança. (E8)

Dentre os aspectos relacionados ao papel do enfermeiro, após a implementação do Protocolo, os participantes do estudo também reconheceram melhoria no atributo longitudinalidade, o que promove nas pessoas com DM uma maior adesão ao tratamento.

Acho que ele [o protocolo] vem para reforçar os atributos da atenção primária, em relação ao acesso, trabalho em equipe e coordenação do cuidado. Porque a gente deixa de focar só no médico, no acompanhamento realizado pelo médico e deixa o paciente livre, com acesso maior para procurar a equipe. Em relação de acesso, as pessoas têm mais um profissional. Antes era só o médico agora ele tem o médico e o enfermeiro, que podem fazer esse tipo de atendimento para ele. (E08)

A longitudinalidade eu não sei se ele chega a influenciar, porque é independente do protocolo. É uma coisa que é da atenção primária então eu acho que ele não vai trazer tanta mudança nisso, mas pode ajudar. $\mathrm{Na}$ coordenação do cuidado ajuda porque o enfermeiro vai passar a fazer parte do cuidado do paciente e eu acho que é isso. (Em2)

Além disso, as solicitações de exames também permitem uma melhor coordenação do cuidado das pessoas com DM. Pois, como enfermeiro atuante nas renovações e solicitações de exames há um reforço na coordenação do cuidado das pessoas com DM, permitindo ao profissional e equipe melhor o acompanhamento e a avaliação da adesão ao tratamento, bem como a necessidade de intervenções de outros profissionais. 
Nossa, está ajudando muito em tudo! Na consulta programática e espontânea a renovação de medicação, só fica de fora o paciente insulinizado. Ajuda muito! Já renovei várias receitas, ajuda muito a facilitar a adesão do paciente. [...] O paciente já tem vínculo, traz exames, mostra para a gente saber se tem que fazer a interconsulta ou não, facilita e agiliza muito o atendimento. (E16)

Os médicos também reconheceram a qualificação dos enfermeiros, identificando suas potencialidades. A aceitação dos médicos a esse Protocolo parece estar vinculada à sua inserção na APS, compreendendo a importância do mesmo e percebendo-o como um avanço na ampliação e qualificação da atenção às pessoas com DM.

Olha, eu acho que o protocolo em si é uma coisa que pode vir a contribuir muito! Eu acho que em Florianópolis a gente tem comparado, não vou dizer como Brasil todo, porque tem muitas realidades diferentes, mas eu tenho tido a oportunidade, às vezes, de acompanhar o trabalho de outros municípios na atenção primária, o trabalho que faço junto ao Ministério. O que eu vejo, assim, a enfermagem de Florianópolis tem um grupo de profissionais muito bem qualificados, já antes até de existir o protocolo. Por isso, talvez, a gente tenha saído nessa quase que iniciativa, não vou dizer que em outros lugares não tenham, mas tentar criar esse protocolo, sistematizar melhor o trabalho da enfermagem, até que ponto a enfermagem consegue ter autonomia para fazer algumas coisas, eu acho que eles vêm muito bem assim. (Em3)

\section{Discussão}

Protocolos para a atenção de pessoas com DM são reconhecidos em diferentes realidades como essenciais para organização e promoção de maior segurança no cuidado, possibilitando uma assistência adequada, eficaz e atualizada. ${ }^{10-12}$ Convergente à essas indicações os resultados do presente estudo, indicaram que o Protocolo contribui com a autonomia e a ampliação da prática clínica aos enfermeiros na atenção às pessoas com DM. 
O município de Florianópolis/SC se constitui como pioneiro na elaboração e implementação de Protocolo de Enfermagem no Estado, incluindo o atendimento com vistas à integralidade do cuidado, o qual agrega a prática de solicitação de exames laboratoriais e prescrição de medicamentos, como atividade legal a esse profissional. Protocolos clínicos são reconhecidos como ferramenta que proporciona maior autonomia e segurança à prática do enfermeiro. ${ }^{7}$

Mesmo que o presente estudo não tenha avaliado os resultados clínicos nas pessoas com DM, pode-se observar uma avaliação favorável dos profissionais enfermeiros e médicos, acreditando que o Protocolo trouxe mudanças clínicas pelo aumento da adesão ao tratamento à essa população. O processo de implantação do Protocolo teve como orientação as metodologias participativas, o que trouxe envolvimento dos atores sociais envolvidos, repercutindo em laços de confiança mútua entre os pares. Essa estratégia de envolvimento dos profissionais é referida como promovendo maior confiabilidade e estimulando a adoção de suas recomendações. ${ }^{13}$ Além disso, o envolvimento dos profissionais pode ter sido um elemento importante para aprofundar o conhecimento acerca dessa condição crônica, uma vez que estudos têm mostrado que esses profissionais em diferentes países têm déficits significativos de conhecimento em muitos aspectos do tratamento do diabetes, e que esse é um problema de longa data e contínuo. ${ }^{11,14}$

A metodologia de discussão de casos clínicos, aplicada durante o treinamento para elaboração do Protocolo foi muito bem avaliada pelos participantes do estudo. Nesse sentido, a utilização de metodologias que utilizam a discussão de casos clínicos, como aprendizado baseado em problemas, tem reconhecimento de sua eficácia na formação na área da saúde. Essa estratégia contribui para minimizar queixas relacionadas às dificuldades do exercício profissional, oferecendo segurança para a atuação clínica. ${ }^{15-16}$ 
Protocolo de enfermagem para o cuidado da pessoa com diabetes mellitus na atenção P... $\mid 14$

Com a implementação do Protocolo houve reconhecimento da ampliação do cuidado de enfermagem às pessoas com DM na APS, a qual esteve diretamente relacionada ao empoderamento desse profissional para executar as condutas descritas no Protocolo, mantendo a compreensão do trabalho em equipe. A capacidade do enfermeiro para ampliação de suas ações está alicerçada na formação em nível de graduação e também de pós-graduação de qualidade e que está voltada para a implementação das políticas de saúde no Brasil. ${ }^{17}$

Apesar dos atendimentos às pessoas com DM já estarem estabelecidos em programas nacionais há algum tempo, ainda são encontradas barreiras nesse acompanhamento, especialmente pela falta de autonomia do enfermeiro para a solicitação de exames e renovações de receitas. $^{18} \mathrm{O}$ Protocolo trouxe autonomia ao enfermeiro, além de fortalecer as ações realizadas nas consultas de enfermagem como uma atribuição da profissão. Essas ações reforçam a importância da integralidade do cuidado às pessoas com DM nesse nível da atenção em saúde e reforçam condutas que trazem benefícios à qualidade de vida desse público como a prevenção de suas complicações agudas e crônicas.

Considerando a relevância do cuidado integral é reconhecida a existência de lacunas no mesmo, mesmo em países desenvolvidos como o Estados Unidos, com indicação de que diretrizes e protocolos sejam implantados e avaliados, visando o desenvolvimento de uma assistência em saúde que considere as necessidades das pessoas com DM. ${ }^{1}$ Alguns estudos têm mostrado a necessidade de dar atenção a formação dos enfermeiros em relação ao cuidado de pessoas com DM, aliados à outras ações que ajudem a minimizar a pesada carga de trabalho que enfrentam. Ressaltam ainda, a importância de realizar pesquisas no próprio local de trabalho. ${ }^{11,13}$

O Protocolo possibilitou uma reorganização do processo de trabalho nessas equipes, sistematizando o atendimento de enfermagem a essa população. Promoveu ainda o exercício de ações, tais como a prescrição de medicamentos e a solicitação de exames, já estabelecidas na legislação brasileira, porém ainda não muito difundidas no Brasil em relação ao cuidado de 
pessoas com DM. Na Lei do Exercício Profissional dos enfermeiros estabelece que este pode realizar a prescrição de medicamentos, quando constantes em programas de saúde pública e rotinas aprovadas pela instituição. Em relação à solicitação de exames de rotina e complementares, o enfermeiro pode solicitá-los no exercício de suas atividades profissionais. Portanto, essas ações são respaldadas legalmente e pertencem ao escopo de atribuições do enfermeiro. ${ }^{19}$

Consoante à perspectiva dos enfermeiros, os médicos, também consideraram que o Protocolo contribuiu para autonomia do enfermeiro, o fortalecimento do vínculo, do acesso, da resolutividade e da coordenação do cuidado, sendo essencial no cuidado às pessoas com DM, e converge com os atributos da Política Nacional de Atenção Básica. ${ }^{8}$ A introdução do Protocolo na dinâmica de trabalho possibilitou um fortalecimento das interconsultas e da relação entre médico e enfermeiro, com o aumento das discussões de casos clínicos e compartilhamento do cuidado, trazendo novas forma de alcance de ações pactuadas. $\mathrm{O}$ apoio do profissional médico da equipe, relacionado aos protocolos de enfermagem, fortalece a interdisciplinaridade no processo de trabalho. ${ }^{20}$

Uma demanda excessiva foi identificada como fator negativo nos atendimentos dos enfermeiros. O uso de protocolos, bem como melhores condutas, podem ser prejudicadas quando há um excesso de demanda de atendimento para esse profissional. $O$ aumento de cargas de trabalho na APS e o excesso de demanda são aspectos compreendidos como responsáveis pela insatisfação dos profissionais de saúde no ambiente de trabalho. ${ }^{2,21}$

Há uma objeção quanto à aceitação das prescrições do enfermeiro em farmácias privadas, mesmo regulamentadas pelo Ministério da Saúde. ${ }^{22}$ Essa situação é limitante, especialmente nos casos de falta provisória de medicamentos na rede pública, necessitando de atendimento médico para receberem prescrição. Portanto, é necessário um avanço em âmbito nacional 
relacionado à aceitação da prescrição de enfermagem respaldadas por protocolos clínicos, nos serviços de saúde.

Nesse estudo, os participantes reconheceram que o uso do Protocolo fortaleceu a segurança no cuidado às pessoas com DM, visto que os enfermeiros relataram uma maior confiança em seus atendimentos a essa população, com o favorecimento de melhores condutas à essa demanda. A padronização da assistência é outro fator de segurança apontado, possibilitando uma conduta homogênia entre a categoria, bem como entre o próprio binômio médico-enfermeiro, entendendo que o Protocolo está de acordo com o Guia Prático para Médicos e Enfermeiros utilizados no município. ${ }^{4,6-8}$

A forma de apresentação e de acesso ao Protocolo, também responde as necessidades de sua utilização na prática. A objetividade do material que pode ser acessado durante um atendimento possui relevância para que seu uso seja viável e incorporado na prática. A facilidade de uso do protocolo e seu fácil acesso são considerados pontos positivos na avaliação de protocolos. $^{6-7,23}$

Os enfermeiros reconhecem que estão promovendo maior adesão ao tratamento das pessoas com DM. Isso pode estar relacionado à consolidação da longitudinalidade. O vínculo estabelecido com essa população por meio do reconhecimento do trabalho na APS parece ser um elemento propulsor dessa situação, constituindo-se os princípios e diretrizes na Política Nacional de Atenção Básica. ${ }^{8,24}$

\section{Conclusão}

Avaliar a contribuição do Protocolo de Enfermagem - Volume I, para o cuidado à saúde de pessoas com DM, na perspectiva de enfermeiros e médicos que atuam na APS de Florianópolis/SC, permitiu reconhecer que além de cumprir com seu papel de padronizador de 
condutas, guiado pelas evidências científicas, o Protocolo também contribuiu para a qualificação do cuidado de enfermagem. Essa avaliação favorável está sustentada por argumentos que mostram que o mesmo tem reconhecimento e confiança da categoria em relação à qualidade do conteúdo, pertinência e aplicabilidade.

Com relação à atuação do enfermeiro, o mesmo possibilitou autonomia profissional e respaldo para execução dos princípios e diretrizes da Política Nacional de Atenção Básica, tais como o fortalecimento do vínculo, longitudinalidade, coordenação do cuidado, resolutividade e melhoria da acessibilidade, por meio da ampliação da clínica. Além disso, o Protocolo contribuiu para melhorar o cuidado de enfermagem em relação à sua prática clínica, com ações mais sustentadas teoricamente e coordenadas com outros profissionais da equipe.

Como limitações o estudo avaliou a contribuição do Protocolo de Enfermagem somente na perspectiva dos médicos e enfermeiros, sugerindo que outras pesquisas sejam desenvolvidas incluindo gestores e usuários, com avaliação clínica das pessoas atendidas após sua implementação, ampliando-se assim a avaliação do protocolo em questão.

\section{Referências}

1. American Diabetes Association (ADA). Classification and diagnosis of diabetes: standards of medical care in diabetes-2019. Diabetes Care; 2019;42(Suppl 1):S13-S28.

2. Salci MA, Meirelles BHS, Silva DMGV. Health education to prevent chronic diabetes mellitus complications in primary care. Esc Anna Nery Rev Enferm. 2018;22(1). doi: http://dx.doi.org/10.1590/2177-9465-ean-2017-0262

3. Conselho Regional de Enfermagem de São Paulo. Guia para construção de protocolos assistenciais de enfermagem. São Paulo (SP): COREN-SP; 2015 [acesso em 2019 nov 11]. Disponível em: https://portal.coren-sp.gov.br/sites/default/files/Protocolo-web.pdf

4. Almeida ER, Moutinho CB, Carvalho SAS, Araújo MRN. Report about the construction of a nursing protocol in child care in primary care. Rev Enferm UFPE On Line [Internet]. 2016 [cited 2019 nov 11];10(2):683-91. Available from: https://periodicos.ufpe.br/revistas/revistaenfermagem/article/view/11006 
Protocolo de enfermagem para o cuidado da pessoa com diabetes mellitus na atenção P... $\mid 18$

5. Prefeitura Municipal de Florianópolis. Secretaria Municipal de Saúde de Florianópolis. Protocolos de Enfermagem. Volume 1: hipertensão, diabetes e outros fatores associados a doenças cardiovasculares [Internet]. Florianópolis: Secretaria Municipal da Saúde; 2015 [acesso em 2019 nov 11]. Disponível em: http://www.pmf.sc.gov.br/arquivos/arquivos/pdf/03_01_2020_13.13.01.d79094d25246fcb4b4a021e63dc27a7 d.pdf

6. Gubert FA, Santos DAS, Pinheiro MTM, Brito LLMS, Pinheiro SRCS, Martins MC. Development of a nursing protocol for childcare consultations. Rev Rene (Online). 2016;16(1). doi: http://dx.doi.org/10.15253/2175-6783.2015000100011

7. Vieira AC, Bertoncello KCG, Girondi JBR, Nascimento ERP, Hammerschmidt KSA, Zeferinho MT. Perception of emergency nurses in using a chest pain assessment protocol. Texto \& Contexto Enferm. 2016;25(1)e1830014. doi: https://doi.org/10.1590/0104-07072016001830014

8. BRASIL. Ministério da Saúde. Portaria no 2.436, de 21 de setembro de 2017. Aprova a Política Nacional de Atenção Básica, estabelecendo a revisão de diretrizes para a organização da Atenção Básica, no âmbito do Sistema Único de Saúde (SUS). Brasília, DF: Ministério da Saúde, 2017. Disponível em: http://www.brasilsus.com.br/index.php/legislacoes/gabinete-do-ministro/16247-portaria-n-2-436-de21-de-setembro-de-2017. Acesso em: 11 nov. 2019.

9. Hsieh HF, Shannon SE. Three approaches to qualitative content analysis. Qual Health Res. 2005;15(9):1277-88. doi: https://doi.org/10.1177/1049732305276687

10. Arnold P, Scheurer D, Dake AW, Hedgpeth A, Hutto A, Colquitt C, et al. Hospital guidelines for diabetes management and the joint commission-american diabetes association inpatient diabetes certification. Am J Med Sci [Internet]. 2016 [cited 2020 mar 31];351(4):333-41; Available from: https://www.amjmedsci.org/article/S0002-9629(15)38014-9/pdf

11. Sang D, Huang Y-P, Cheng X-Q. Quantitative and qualitative investigation of traditional Chinese medicine nursing protocols for Type 2 Diabetes Mellitus. J Integr Nurs. 2019;1(2):80-5. doi: https://doi.org/10.35437/intnur.issn.2663-4481.2019.01.02.03

12. Bagweneza V, Musabirema P, Mwiseneza MJ, Collins A, Bhengu BR. Diabetes health education: nurses' knowledge of essential components at a Rwandan hospital. Rwanda J Med Health Sci;2019;2(2):172-77. doi: http://dx.doi.org/10.4314/rjmhs.v2i2.13

13. Mega TP, Lopes ACF, Santos VCC, Petramale CA. Protocolos clínicos e diretrizes terapêuticas no SUS: histórico, desafios e perspectivas. Rev Gest Saúde (Brasília) [Internet]. 2015 [acesso em 2019 nov 2011];6(4):3275-85. Disponível em: https://periodicos.unb.br/index.php/rgs/article/view/3333

14. Alotaibi A, Al-Ganmi A, Gholizadeh L, Perry L. Diabetes knowledge of nurses in different countries: an integrative review. Nurse Educ Today. 2016;39:32-49. doi: http://dx.doi.org/10.1016/j.nedt.2016.01.017

15. Pinto ML, Mistro FZ, Uemura ST. Ensino baseado em problemas como prática pedagógica aplicada 
a alunos ingressantes no curso de Odontologia. Rev ABENO. 2016;16(3):28-35. doi: https://doi.org/10.30979/rev.abeno.v16i3.278

16. Anjos RMP, Rodriguez JW, Fujiki RHM, Bernardo MO. Aprendizagem baseada em equipe: uma estratégia de ensino aplicada na área da imaginologia. 33- Congresso da SUMEP. 2016 out 03; Sorocaba, BR. (Rev Fac Cienc Med Sorocaba [Internet]. 2016 [acesso em 2019 nov 11];18(Supl):90. Disponível em: https://revistas.pucsp.br/index.php/RFCMS/article/view/29849)

17. Winters JRF, Prado MLD, Heidemann ITSB. Nursing education oriented to the principles of the Unified Health System: perception of graduates. Esc Anna Nery Rev Enferm [Internet]. 2016 [cited 2019 nov 11];20(2). Available from: https:/www.scielo.br/scielo.php?pid=S1414$81452016000200248 \&$ script $=$ sci_arttext\&tlng=en

18. Alves KYA, Salvador PTCO, Tourinho FSV, Santos VEP. Analysis of the concept "nursing protocols" from the evolutionary vision of rodgers. Rev Enferm UFPE On Line [Internet]. 2014 [cited 2019 nov 11];8(1):177-82. Available from: https://periodicos.ufpe.br/revistas/revistaenfermagem/issue/view/1235

19. CONSELHO FEDERAL DE ENFERMAGEM (BR). Resolução 271/2002. Regulamenta as ações do enfermeiro na consulta, prescrição de medicamentos e requisição de exames. Rio de Janeiro (RJ): COFEN; 2002.

20. Oliveira BN, Alves EAS, Araújo CA, Santos ALB, Souza Neto FDCV, Maia Neto JP. Saúde do homem na Atenção Primária à Saúde: reflexões acerca da multiplicidade de olhares na avaliação corporal. Rev Baiana Saúde Pública. 2015;38(3):751. doi: https://doi.org/10.22278/23182660.2014.v38.n3.a638

21. Lima L, Pires DEP, Forte ECN, Medeiros F. Job satisfaction and dissatisfaction of primary health care professionals. Esc Anna Nery Rev Enferm [Internet]. 2014 [cited 2019 nov 11];18(1). Available from: http://dx.doi.org/10.5935/1414-8145.20140003

22. Martiniano CS, Andrade PS, Magalhães FC, Souza FF, Clementino FS, Uchôa SAC. Legalization of nurse prescribing of medication in Brazil: history, trends and challenges. Texto \& Contexto Enferm. 2015;24(3):809-17. doi: http://dx.doi.org/10.1590/0104-07072015001720014

23. Bohn MLS, Lima MADS, Duro CLM, Abreu KP. Nurses' perception on the use of the manchester risk classification system protocol. Ciênc Cuid Saúde. 2015;14(2):1004. doi: http://dx.doi.org/10.4025/cienccuidsaude.v14i2.21359

24. Romanoski PJ, Silva DMGV, Böell JEW, Guanilo MEE, Rocha FL, Campos TSP. Saberes e práticas dos profissionais de saúde na atenção à pessoa com Diabetes Mellitus. Rev Enferm UFSM. 2018;8(4):688-701. doi: http://dx.doi.org/10.5902/2179769230931 


\section{Autor correspondente}

Priscylla Lauterte

E-mail: pry.enfermeira@gmail.com

Endreço: Rua Princesa Isabel, 165 - Forquilhinhas, São Jose/SC

CEP: 88106730

\section{Contribuições de Autoria}

\section{1 - Priscylla Lauterte}

Construção do projeto, condução da coleta e análise dos dados, bem como revisão crítica da versão final do relatório de pesquisa.

\section{2 - Denise Maria Vieira Guerreiro da Silva}

Construção e planejamento do projeto de pesquisa, participação da análise e interpretação dos dados e da redação do texto.

\section{3 - Maria Aparecida Salci}

Participação da análise dos dados, bem como revisão crítica da versão final do relatório de pesquisa.

\section{4 - Ivonete Teresinha Schulter Buss Heidemann}

Revisão crítica da versão final do relatório de pesquisa.

\section{5 - Priscila Juceli Romanoski}

Revisão crítica da versão final do relatório de pesquisa.

\section{Como citar este artigo}

Lauterte P, Silva DMVG, Salci MA, Heidemann ITSB, Romanoski PJ. Protocolo de enfermagem para o cuidado da pessoa com diabetes mellitus na atenção primária. Rev. Enferm. UFSM. 2020 [Acesso em: Anos Mês Dia]; vol.10 e72: 1-20. DOI:https://doi.org/10.5902/2179769240638 